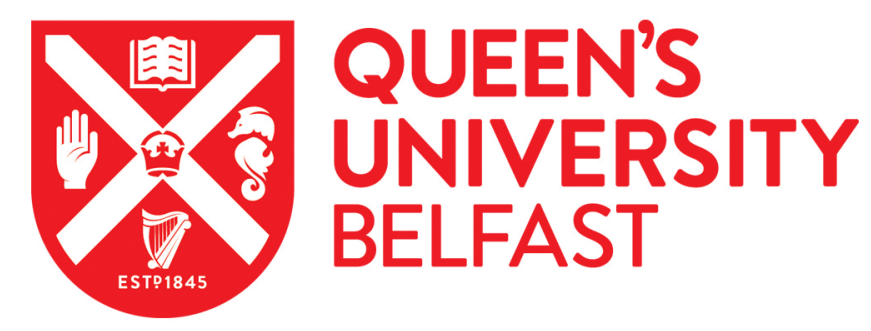

\title{
Perceptual Learning of Acoustic Noise by Individuals With Dyslexia
}

Agus, T. R., Carrión-Castillo, A., Pressnitzer, D., \& Ramus, F. (2014). Perceptual Learning of Acoustic Noise by Individuals With Dyslexia. JOURNAL OF SPEECH LANGUAGE AND HEARING RESEARCH, 57, 1069-1077. https://doi.org/10.1044/1092-4388(2013/13-0020)

Published in:

JOURNAL OF SPEECH LANGUAGE AND HEARING RESEARCH

\section{Document Version:}

Peer reviewed version

Queen's University Belfast - Research Portal:

Link to publication record in Queen's University Belfast Research Portal

\section{Publisher rights}

Copyright 2016 ASHA

This is an open access article published under a Creative Commons Attribution-NonCommercial-NoDerivs License

(https://creativecommons.org/licenses/by-nc-nd/4.0/), which permits distribution and reproduction for non-commercial purposes, provided the author and source are cited.

\section{General rights}

Copyright for the publications made accessible via the Queen's University Belfast Research Portal is retained by the author(s) and / or other copyright owners and it is a condition of accessing these publications that users recognise and abide by the legal requirements associated with these rights.

Take down policy

The Research Portal is Queen's institutional repository that provides access to Queen's research output. Every effort has been made to ensure that content in the Research Portal does not infringe any person's rights, or applicable UK laws. If you discover content in the Research Portal that you believe breaches copyright or violates any law, please contact openaccess@qub.ac.uk. 


\section{Perceptual Learning of Acoustic Noise by Dyslexic Individuals}

\begin{tabular}{|r|l|}
\hline Journal: & Journal of Speech, Language, and Hearing Research \\
\hline Manuscript ID: & JSLHR-H-13-0020.R2 \\
\hline Manuscript Type: & Research Article \\
\hline Date Submitted by the Author: & 26-Aug-2013 \\
\hline Complete List of Authors: & $\begin{array}{l}\text { Agus, Trevor; CNRS \& Laboratoire de Psychologie de la Perception \& École } \\
\text { normale supérieure, Institut d'études cognitives, Equipe audition } \\
\text { Carrión-Castillo, Amaia; École Normale Supérieure, CNRS, EHESS, } \\
\text { Laboratoire de Sciences Cognitives et Psycholinguistique } \\
\text { Pressnitzer, Daniel; CNRS \& Laboratoire de Psychologie de la Perception \& } \\
\text { École normale supérieure, Institut d'études cognitives, Equipe audition } \\
\text { Ramus, Franck; École Normale Supérieure, CNRS, EHESS, Laboratoire de } \\
\text { Sciences Cognitives et Psycholinguistique }\end{array}$ \\
\hline Keywords: & \begin{tabular}{l} 
Developmental disorders, Memory, Hearing, Cognition, Psychoacoustics \\
\hline
\end{tabular} \\
\hline
\end{tabular}




\title{
Perceptual Learning of Acoustic Noise by Dyslexic Individuals
}

\author{
Trevor R. Agus ${ }^{1}$, Amaia Carrión-Castillo ${ }^{2}$, Daniel Pressnitzer ${ }^{1}$, and Franck Ramus ${ }^{2}$ \\ ${ }^{1}$ CNRS \& Laboratoire de Psychologie de la Perception \& École normale supérieure \\ ${ }^{2}$ Laboratoire de Sciences Cognitives et Psycholinguistique \& École normale supérieure \\ \& CNRS \& EHESS
}

\begin{abstract}
Correspondence concerning this article should be addressed to Trevor Agus, CNRS \& Laboratoire de Psychologie de la Perception \& École normale supérieure, 29 rue d'Ulm, 75230 Paris Cedex 5, France
\end{abstract}

Phone number: +33144322673

Fax number: +3314432 2686

Email address: trevor.agus@ens.fr

Running Title: Dyslexia and the learning of noise

Date: $26^{\text {th }}$ August 2013 


\section{ABSTRACT}

Purpose: A phonological deficit is thought to affect most individuals with developmental dyslexia. The present study addresses whether the phonological deficit is caused by difficulties with perceptual learning of fine acoustic details.

Method: A demanding test of non-verbal auditory memory, "noise learning”, was administered to both dyslexic and control adult participants. On each trial, listeners had to decide whether a stimulus was a 1-s noise token or two abutting presentations of the same 0.5-s noise token (repeated noise). Without the listener's knowledge, the exact same noise tokens were presented over many trials. An improved ability to perform the task for such "reference" noises reflects learning of their acoustic details.

Results: Dyslexic listeners did not differ from controls in any aspect of the task, qualitatively or quantitatively: they required the same amount of training to achieve discrimination of repeated from non-repeated noises; they learnt the reference noises as often and as rapidly as the control group. Yet they showed all the hallmarks of dyslexia, including a well-characterized phonological deficit.

Conclusions: Our data do not support the hypothesis that deficits in basic auditory processing, or non-verbal learning and memory, are the cause of the phonological deficit in dyslexia.

Keywords: Developmental dyslexia; Perceptual learning; Phonological deficit. 
Perceptual Learning of Acoustic Noise by Dyslexic Individuals

Developmental dyslexia is a neurodevelopmental disorder that specifically disrupts the acquisition of reading and spelling, which affects about 3-7\% of school children (Lindgren, De Renzi, \& Richman, 1985; World Health Organization, 2011). Dyslexia cannot be attributed to uncorrected sensory disorders, low intelligence or educational deprivation. Rather, research carried out over the last 40 years has provided remarkably convergent evidence in support of the general idea that the cognitive basis of dyslexia is a deficit in phonological representations and/or processing, at least for a majority of dyslexic children (Dole, Hoen, \& Meunier, 2012; Ramus, 2003; Vellutino, Fletcher, Snowling, \& Scanlon, 2004). This deficit may have a causal role in the development of dyslexia (Guttorm et al., 2005; Leppänen et al., 2010). Nevertheless, there are a number of open questions.

One question has to do with the specific nature of the phonological deficit. Dyslexic individuals show particularly poor performance in tasks that fall broadly into three categories (Wagner \& Torgesen, 1987). These task categories are: 1) phonological awareness (tasks that require conscious attention to and manipulation of individual speech sounds); 2) verbal short-term memory and verbal working memory (tasks that require perception, storage for a few seconds, and optionally production of verbal material); 3) rapid automatized naming (tasks that require the rapid retrieval and naming of a series of objects, colors or digits). Because each of these three task categories involves phonological representations, a widely accepted hypothesis is that the representations themselves are in some way degraded in dyslexic individuals. However, 
poor performance is always observed in complex tasks, which involve various combinations of conscious attention, verbal memory, analytic skills and linguistic abilities. An alternative hypothesis is therefore that the phonological representations are intact, but that access to them might be impaired in certain cognitive operations (Ramus \& Szenkovits, 2008). Data that would allow adjudication between these two hypotheses remain scarce (Ramus \& Ahissar, 2012).

Another question is whether the phonological deficit is specific to speech sounds, or whether it reflects a more general auditory processing impairment. Here again the evidence is mixed. While a considerable body of data suggests that some dyslexic individuals do have auditory deficits, there is considerable disagreement over the nature of these deficits (Amitay, Ahissar, \& Nelken, 2002; Farmer \& Klein, 1995; Goswami, Gerson, \& Astric, 2010; Goswami et al., 2011; Hornickel, Chandrasekaran, Zecker, \& Kraus, 2012; Tallal, 1980). There is also convincing evidence that auditory deficits affect only a subset of the dyslexic population, and that their relationship with the phonological deficit and reading disability is weak if reliable at all (Banai \& Ahissar, 2006; Ramus et al., 2003; Rosen, 2003; White, Frith et al., 2006; White, Milne et al., 2006). Alternative interpretations have again been proposed, suggesting that performance differences on tasks hypothesized to tap into basic auditory processing may, in dyslexic individuals, reflect variance in cognitive abilities such as auditory attention, learning, memory, and metacognition (Ahissar, Lubin, Putter-Katz, \& Banai, 2006; Ramus \& Ahissar, 2012). Thus, while it seems plausible that auditory deficits could be the cause of a readingrelevant phonological deficit, the evidence remains inconclusive. 
As a consequence, recent theories implicate cognitive deficits. For instance, the "anchoring"-deficit theory postulates that dyslexic individuals have difficulties in detecting and exploiting regularities in the perceptual stream. For instance, failing to recognize that a sound is re-occurring many times would force dyslexics to spend the same cognitive resources for each of its appearances and impair task performance (Ahissar, 2007). The anchoring deficit can be assessed by contrasting two variants of an AX discrimination task: either with both stimuli varied randomly from trial to trial, or with one stimulus kept constant across all trials. Control participants typically benefit from the presence of a constant stimulus, forming an "anchor" that spares their short-term memory. Dyslexic participants do not (Ahissar et al., 2006; Oganian \& Ahissar, 2012). The anchoring deficit is presumed to be modality-independent. Another similar but distinct recent hypothesis is that dyslexic individuals have difficulties learning serialorder information, whether in the verbal, auditory or visual modality (Szmalec, Loncke, Page, \& Duyck, 2011).

Both of these new theories and the questions raised by Ramus and Szenkovits (2008) on the nature of the phonological deficit warrant a reconsideration of the role of attention, learning and memory in dyslexia. In this context, the present study explores the hypothesis that dyslexic individuals have deficits in learning the acoustic details of new sounds.

Memory underlies a wide range of auditory tasks, even basic ones such as temporal masking (see Demany \& Semal, 2008 for a review). As such, deficits in auditory learning, when applied to speech sounds, could potentially account for difficulties in phonological awareness and verbal short-term memory. In order to test 
non-verbal auditory memory, the present study builds on a paradigm developed by Agus, Thorpe, and Pressnitzer (2010) and Agus and Pressnitzer (2013).

Agus et al. (2010) used white noise to observe behaviorally the emergence of new auditory memories. The waveform of white noise can be generated as a long series of random numbers with a Gaussian distribution. It is guaranteed that a specific token of white noise, generated in the laboratory, has never been heard before by listeners. Furthermore, as white noise is fully unpredictable and as its details are impossible to rehearse mentally, such a stimulus provides arguably a difficult test of pure non-verbal auditory learning. Figure 1 shows a schematic of the task used here and in Agus et al. (2010). Listeners were first trained to report within-trial repetitions in noises: when a half-second noise is presented repeatedly, these repetitions can be perceived, for example, as a "whooshing" pattern (Guttman \& Julesz, 1963). The task set to listeners was to distinguish noises that contained repetitions from continuous noises of the same duration. Then, on subsequent blocks for which listeners continued to perform the same task, one arbitrary exemplar of the repeated noise (the "reference repeated noise"; RefRN) was presented identically in multiple trials. The RefRN token, which was thus repeated both within and across trials, was interspersed in random positions amongst trials containing only within-trial repetitions (repeated noise, $\mathrm{RN}$ ), or no repetition at all (N). Although the listeners had not been told about the RefRN and were not aware that learning would be beneficial to their performance, they reported the RefRNs as repeated much more reliably than the RNs they only heard once. Furthermore, once learnt, the RefRN token could be recognized even without any within-trial repetition (Agus and Pressnitzer, 2013). This 
shows that listeners can learn at least some of the specific acoustic details of a 0.5 -s noise token.

- Insert Figure 1 around here-

The Agus et al. (2010) study provides a psychophysical task showing that even completely arbitrary complex sounds can be learnt by naive listeners. Learning was unsupervised, occurring in only a few exposures, and the memory traces were longlasting. The task therefore measures a set of skills that could underlie many aspects of auditory performance and development, including speech acquisition and perception. It seems relevant to ask to what extent dyslexic listeners can perform such a noise-learning task. Indeed, under the hypothesis that auditory attention, learning or memory skills may be impaired in dyslexia, lower performance would be predicted. Under the hypothesis of a basic auditory processing disorder, performance on noise learning would also be predicted to be impaired, as the representation to be learned would be somehow impoverished or more variable (even though this paradigm is not particularly designed to specifically test the popular hypothesis of a rapid auditory processing disorder; Tallal, 1980). Conversely, if all of these aspects of auditory processing are unaffected in dyslexia, normal performance would be predicted. The present study therefore aims to test these hypotheses by administering Agus et al.'s (2010) sound-learning experiment to matched groups of adult dyslexic and control participants.

\section{METHODS}

Eighteen adult dyslexic participants ( 9 male, 9 female) and eighteen control participants (8 male, 10 female) with similar academic backgrounds, ages and non-verbal 
IQs took part in this study. They were recruited through advertisements in Parisian universities and received $€ 15$ per hour of participation. Inclusion criteria were (1) to be a native, monolingual speaker of French aged 18 years old or over, (2) to report no known neurological/psychiatric disorders or hearing impairment, and (3) to have a non-verbal IQ above 80 . For controls, the crucial criteria were (4a) to report no known history of reading/oral language difficulties, and to have a reading age above the ceiling $\left(8^{\text {th }}\right.$ grade; 13 years old) of our standardized reading test. For dyslexics, (4b) a history of reading difficulties at school age, a reading fluency score below the level of $8^{\text {th }}$ grade. Participants underwent a diagnostic battery during a preliminary session to ensure that they met inclusion criteria. Then the experimental tests took place in a separate session. Ethical approval was granted by the local ethics committee (CPP Bicêtre).

\section{Diagnostic procedure}

The diagnostic battery included intelligence and reading tests for the purpose of inclusion criteria. In addition, it included a set of classic phonological tasks in which dyslexic individuals typically show poor performance.

Non-verbal intelligence. Non-verbal intelligence was assessed by using either Raven's Advanced Progressive Matrices Set I and Set II (Raven, Raven, \& Court, 1998) or the Picture completion and Matrices subtests of the Wechsler Adult Intelligence Scale (Wechsler, 2000).

Reading skills. Reading skills were assessed by the standardized French reading test "L'alouette" (Lefavrais, 1967). This meaningless text comprises 265 words ranging from common to rarely used words. Participants are instructed to read the text as fast and as accurately as possible. Standardized reading fluency scores are computed by 
combining total reading time and reading errors (Gola-Asmussen, Lequette, Pouget, Rouyer, \& Zorman, 2010).

Orthographic skills. Orthographic skills were assessed with a speeded forcedchoice task. Participants were presented successively with 24 triplets of words on computer screen. Each triplet included a correctly spelt word, and two misspelled versions. Participants had to press as quickly as possible the key corresponding to the correct spelling. Scores are the number of correct trials per second.

Digit spans. Forward and backward digit spans (from the French version of WAIS-III; Wechsler, 2000) were used to compute age-appropriate scaled scores, to obtain a measure of phonological working memory.

Spoonerisms. Participant were verbally presented with pairs of words and were instructed to swap the first sound of the two words, then pronounce the resulting pseudowords while maintaining their correct order. A composite score taking into account both accuracy and speed is computed.

Rapid Automatized Naming (RAN). Participants completed three versions: picture and digit naming ( 2 sheets of 50 objects or digits) adapted from the Phonological Assessment Battery (Frederickson, Frith, \& Reason, 1997), and color naming (2 sheets of 50 colors). Each naming test was administered twice with different sheets. The score is the sum of total naming time for both sheets. A composite RAN $z$-score was obtained by averaging $z$-scores from the three RAN tests.

\section{Noise stimuli}

All stimuli were formed from white noise, generated as independent normally distributed random numbers for each token, at a rate of $44.1 \mathrm{kHz}$ and bit depth of 24 bits. 
For repeated noises $(\mathrm{RN})$, a half-second noise token was generated and then concatenated to itself to generate a noise with $2,3,4$, or 10 repeats, depending on the condition. There were no intervening silences at the points of concatenation, and no other local acoustical cues to the repetition. Unrepeated noises $(\mathrm{N})$ were generated as a single token of white noise with the same duration as the RNs (i.e., 1, 1.5, 2 or 5 seconds, depending on the number of repetitions).

In the test blocks, a "reference" repeated noise (RefRN) was generated by exactly the same method as one of the RNs. Thus the difference between an RN and a RefRN lay not in the stimuli themselves, but in their use: each $\mathrm{RN}$ was presented on a single trial then never presented again, whereas exactly the same RefRN stimulus was presented many times (20 trials so 40 presentation of the noise sample) throughout a block. See Figure 1 for a schematic representation of the task.

Different RefRNs were presented in different blocks. In order to minimize between-participant variability, all listeners were presented with the same six RefRN stimuli in their six test blocks. These RefRNs were selected at random.

\section{Procedure}

Listeners launched each trial by pressing a key. After a 1.2-second delay, a noise was presented. Listeners were asked whether they thought the noise was repeated or not. They responded by pressing a key, which triggered the next trial until the end of the block.

Listeners first completed "training" blocks that consisted of RN and N stimuli, without any RefRNs. Initially listeners were asked to distinguish relatively long stimuli (10 repetitions for the $\mathrm{RN}$ ). Over subsequent blocks, the number of repetitions was 
gradually reduced until listeners were distinguishing once-repeated RNs from Ns. Table 1 summarizes the ordering of blocks, numbers of repetitions, and numbers of trials. In all blocks, half the stimuli were repeated and half were unrepeated, interleaved in a random order.

- Insert Table 1 about here-

Then, listeners completed longer "test" blocks, in which half of the repeated noises were RefRN. Listeners were not told about the inclusion of the RefRN stimulus. The ordering of the trials was pseudorandom, with the sole limitation that RefRN stimuli could not be presented on two consecutive trials. This was intended to stretch the duration of auditory memory required to learn the RefRN stimuli. Listeners completed six such blocks, with the same six RefRN for each listener ${ }^{1}$ but with the blocks in random orders.

Listeners received feedback on each response in the training blocks, but not in the test blocks. Thus any learning of the RefRN was unsupervised. Both training blocks and test blocks were completed within a single one-hour session $(M=45$ minutes $)$, distinct from the diagnostic session (see below).

\section{Apparatus}

Listeners were tested individually in a double-walled IAC sound booth, and responded through a computer keyboard. Stimuli were presented through an RME Fireface UC sound-card at a 24-bit resolution and a $44.1 \mathrm{kHz}$ sample-rate. They were presented to both ears simultaneously through Sennheiser HD 600 headphones at $70 \mathrm{~dB}(\mathrm{~A})$. 


\section{Analysis}

For the diagnostic battery, groups were compared on each variable using one-way ANOVAs.

For the repetition-detection task, mixed-design ANOVAs were performed using the statistical package SPSS. Stimulus-related effects $(2,3,4$, or 10 repetitions; N, RN, or RefRN stimuli) were treated as within-subject factors. Group effects (dyslexics vs.

controls) were treated as between-subjects factors. All interactions were included in the resulting mixed-effects models, and type III sums of squares were used throughout.

Greenhouse-Geisser corrections were applied where appropriate.

For the test blocks, where there were a larger number of trial, we estimated the average $d^{\prime}$ sensitivity (Macmillan \& Creelman, 2005) to RN and RefRN for individual participants. These were calculated as

$$
\begin{aligned}
d_{\text {RefRN }}^{\prime} & =z\left(\bar{H}_{\text {RefRN }}\right)-z\left(\bar{F}_{\mathrm{N}}\right) \\
d_{\mathrm{RN}}^{\prime} & =z\left(\bar{H}_{\mathrm{RN}}\right)-z\left(\bar{F}_{\mathrm{N}}\right)
\end{aligned}
$$

where $\bar{H}_{\text {RefRN }}$ and $\bar{H}_{\mathrm{RN}}$ were the mean hit rates for an individual for RefRN and RN stimuli respectively, and $\bar{F}_{\mathrm{N}}$ was the mean false-alarm rate for the $\mathrm{N}$ stimuli. Thus, when the hit-rates are similar to the false-alarm rates, $d^{\prime}$ is close to zero. Greater values of $d^{\prime}$ imply greater sensitivity to the repetitions. An overall criterion, $c$, was also calculated as

$$
c=-\frac{1}{2}\left(z\left(\frac{\bar{H}_{\mathrm{RefRN}}+\bar{H}_{\mathrm{RN}}}{2}\right)+z\left(\bar{F}_{\mathrm{N}}\right)\right)
$$


When listeners report as many stimuli to be repeated as unrepeated, $c$ will be approximately zero; more repetitions reported would lead to a positive $c$ and fewer, to a negative $c$.

Previous results have shown that not all RefRN stimuli are learnt (Agus et al., 2010). Where it was necessary to distinguish blocks in which learning occurred, this was based on whether the listener reported a repetition significantly more in RefRN than for $\mathrm{RN}$ according to a one-tailed Fisher exact test. No correction for multiple comparisons was applied, and as such, it would be expected that a small number of blocks would be incorrectly categorized as showing learning. The equivalent opposite-tailed Fisher exact test was also calculated and we also report the number of blocks with significantly fewer RefRN hits than RN hits. If the apparent learning was due to chance variability alone, the number of blocks with apparent differences in each direction should be roughly equal.

\section{Results}

\section{Diagnostic battery}

Table 2 shows a summary of participants' demographic characteristics and performance on the diagnostic battery. One-way ANOVAs show that participants did not differ in terms of age $\left(F_{1,35}<1, p>.32\right)$ and non-verbal IQ $\left(F_{1,35}=1.5, p=.22\right)$, but differed on all other variables (reading fluency: $F_{1,35}=111.6, p<.001$; orthographic choice: $F_{1,35}=24.3, p<.001$; RAN composite: $F_{1,35}=44.9, p<.001$; digit span: $F_{1,35}=15.8, p<.001$; spoonerisms: $\left.F_{1,35}=15.7, p<.001\right)$. Thus this group of dyslexic participants showed the typical combination of reading and phonological difficulties. 


\section{Training phase}

Figure 2 shows the average hit rates for $\mathrm{RN}$ and false-alarm rates for $\mathrm{N}$ for both groups of listeners with the various numbers of repetitions used in the training block. From ten to three repetitions, there was little change in listeners' hit rates and falsealarms. Thus any putative increased difficulty of the task, with less of a build-up of the percept of repetitions (Kaernbach, 2004), was largely offset by the benefits of training (smaller numbers of repetition were presented last in the training phase). However, for two repetitions, there was a marked reduction in performance, observed as a drop in the number of hits and the rise in false alarms. The mixed-design ANOVA (4 amounts of repetition $\times 2$ types of stimuli $\times 2$ groups of listeners) on the percentage of repetitions reported confirmed that there was a significant effect of stimulus $\left(F_{1,34}=133.90\right.$, $p<.001)$ and a significant interaction of stimulus and number of repetitions $\left(F_{1.62,54.92}=29.02, p<.001\right)$ as well as a significant main effect of the number of repetitions $\left(F_{2.44,82.79}=5.60, p=.003\right)$. The results for the two groups were very similar in all conditions: there was no effect of group $\left(F_{1,34}=0.77, p=.39\right)$, nor any interactions of group with stimulus and/or number of repetition $(p \geq .42)$. Even where the two groups deviated the most, when there were just two repetitions, there was still no significant group difference (mixed-design ANOVA; 2 types of stimuli $\times 2$ groups of listeners; $\left.F_{1,38}=0.83, p=.37\right)$. Thus dyslexic participants did not show greater difficulty learning the task. 


\section{Test phase}

The mean sensitivity for each group, calculated as mean $d^{\prime}$, is shown in Figure 3. In the absence of any learning, the sensitivity to RefRN would be expected, on average, to be the same as the sensitivity to RN. However, overall the sensitivity to the RefRN was greater than to the RN stimulus, showing that listeners did in fact learn the RefRN. A $2 \times 2$ mixed-design ANOVA on $d^{\prime}$ showed there was a main effect of stimulus $\left(F_{1,34}=43.93, p<.001\right)$, but no effect of group $\left(F_{1,34}=0.33, p=.57\right)$ nor any interaction $\left(F_{1,34}=0.48, p=.49\right)$

\section{- Insert Figure 3 about here-}

Similarly, each listener's criterion was calculated individually. The listeners' criteria were on average greater than zero $\left(t_{35}=2.90, p=.006\right)$, which means that they were conservative about reporting repetitions, reporting fewer repetitions than there were repeated stimuli. The dyslexic listeners' criteria $(M=0.1, S D=0.4)$ were not significantly different from the control group's $\left(M=0.2, S D=0.3 ; t_{34}=0.82, p=.42\right)$.

It is also possible to look at the time-course of learning for each group. Figure 4 shows the average hit rates and false-alarm rates, calculated based on the $n^{\text {th }}$ presentation of each type of stimulus. Initially, the hit rates for the RN and RefRN were similar, as would be expected before learning occurred. However, after just a few presentations of the RefRN stimulus, clear differences emerged. This stemmed partly from an increase in the hit rate of the RefRN, but also partly from a decrease in hit rates to the RN. This difference has been previously been attributed to a criterion-shift effect (Agus et al., 2010): as listeners became more sensitive to RefRNs, they remained as able to distinguish 
$\mathrm{N}$ from $\mathrm{RN}$ in their responses, but were more conservative in their responses, such that the overall proportion of stimuli for which repetitions were reported remained constant.

- Insert Figure 4 about here-

What is not shown by the average statistics is that learning did not occur in all blocks. Rather, the number of hits for RefRN only exceeded those for RN in $33 \%$ of blocks for dyslexic listeners (ignoring the first five RefRN and RN trials), and 29\% for the control group, based on a Fisher exact test without correction for multiple comparisons. This is similar to the $31 \%$ of blocks in which learning was observed by naïve participants in Agus et al. (2010). These blocks were evenly spread throughout the listeners: only three dyslexic and three control listeners showed no RefRN learning, which is reasonably similar to the four non-learning listeners that would be expected from 41 listeners each having a 31\% chance of learning any of six blocks independently.

Time courses of hit rates and false-alarm rates are shown in Figure 5 for the blocks in which learning was observed (top panel) and for the rest of the blocks (bottom panel). Three-parameter exponential functions were fitted to the data and accounted for the variability significantly better than a constant value $(\mathrm{p} \leq .02$; Motulsky $\&$ Christopoulos, 2004) for each condition and each group. For the learnt RefRN stimuli, the half-life ${ }^{2}$ of learning was $3.0 \pm 1.8$ (95\% confidence interval centered on the mean) trials for the dyslexic listeners and $2.2 \pm 1.9$ for the control group (not significantly different; $p=.52)$. 


\section{Test correlations with the diagnostic battery}

Sensitivity to noise repetitions and noise learning were not correlated with any test of the diagnostic battery, neither $d_{\mathrm{RN}}^{\prime}$ nor $d_{\text {RefRN }}^{\prime}\left(\left|r_{34}\right| \leq 0.21, p \geq 0.21\right)$.

\section{Discussion}

First, the results for the control group replicate the main findings of Agus et al. (2010). Naive listeners learnt the RefRNs, reporting their repetitions more reliably than for the RN stimuli that they only heard once each. The learning was rapid, with just a few RefRN trials for the half-life of learning. This learning occurred in an unsupervised fashion: the listeners were not asked to memorize the RefRN, nor were they told that a RefRN would reoccur throughout a block. Even if they guessed the true purpose of the experiment, they would not have been able to distinguish the RefRN from other trials without first developing a memory for it. Furthermore, the learning observed was robust, in that it occurred and was sustained despite intervening $\mathrm{N}$ and $\mathrm{RN}$ trials in which different task-relevant stimuli were presented.

These results, and the results relating to the detection of noise repetitions, were extended to a dyslexic population. In terms of repetition detection, no differences were observed between the dyslexic listeners and the control group. First, dyslexics matched the control group in their responses to noises repeated ten times. Then, as the number of repetitions was reduced to four, three, then two, the dyslexic listeners kept up with the control group in their learning of repetition-detection task, despite the increasing perceptual demands of the task. With just two repetitions, both groups found the task equally difficult, with $d$ 's similar to the 0.5 observed by Agus et al. (2010). 
In terms of perceptual learning, the dyslexic listeners showed no deficit relative to the control group. The average sensitivity of each group to the RefRN was strikingly similar, as was their criterion. This resulted from equally fast learning in both groups, as estimated through the time constants of learning for each group, in a similar proportion of blocks. Although there were some listeners who showed no learning of the RefRN at all, there were equal numbers of these listeners in each group.

Thus the present results unambiguously indicate that adult dyslexic participants show normal performance in this psychophysical paradigm. This suggests that their basic auditory processing is not impaired in such a way as to hinder the perception and representation of the subtle acoustic features that characterize each noise stimulus, and that allow listeners to detect whether a stimulus is repeated or not. Note however that the nature of the acoustic features that are memorized in the noise paradigm is still unclear (Kaernbach, 1993). An alternative interpretation is thus that any degradation of acoustic representation for dyslexic listeners does not impact the availability of features that are useful for noise repetition detection and learning.

Most importantly, the learning curve during the experiment was the same for the two groups, suggesting that the non-verbal auditory memory processes of dyslexic listeners are indistinguishable from controls in a noise-learning task. One potential issue is whether the difficulty of the present task was sufficient to adequately tax dyslexic participants' perceptual and cognitive resources. In fact, this task was very difficult, which is why a progressive training phase (from 10 to 4 to 3 to 2 repetitions) was required. The difficult nature of the task was also reflected objectively in the low $d^{\prime}$ values obtained, averaging 0.7 for RN and 1.3 for RefRN. Conversely, could a floor 
effect have prevented us from observing group differences? The distribution of scores does not indicate a floor effect, and indeed almost all participants succeeded in learning some RefRN stimuli. Thus our data suggest that the task was at the right level of difficulty to tax participants' perceptual and cognitive resources while maintaining their performance significantly above chance and floor effects.

One question that could of course be raised is whether these dyslexic participants are really dyslexic and show a phonological deficit. While it is true that they were selected at the high-performing end of the dyslexic population, both their history and their diagnostic data attest that their reading skills lie well below what is expected from their intellectual level. Furthermore the data also show that they have significant difficulties in phonological tasks tapping phonemic awareness, verbal short-term memory and rapid automatized naming. These individuals' phonological deficit requires an explanation, and it seems that the explanation does not include general difficulties in perceiving, attending to and learning complex sounds. Whether this conclusion can be generalized to populations showing more severe impairments will have to be confirmed in future studies.

Given that all participants were adults, the question may also arise whether they might have had genuine auditory processing impairments in childhood, which were resolved by the time they reached adulthood. However, as pointed out before, this raises the question, why would only auditory impairments resolve with time, yet not the phonological deficit nor the reading disability? Further, such an idea has no experimental support showing that auditory processing impairments would be any more frequent in dyslexic children than adults (Ramus et al., 2003; Ramus, White, \& Frith, 2006). 
As our task includes stimuli that re-occur throughout an experimental block, it is interesting to put the results in the context of the "anchoring" deficit in dyslexia (e.g., Oganian \& Ahissar, 2012). performance. In anchoring tasks, a stimulus also re-occurs throughout a block, and a large difference is observed between dyslexics and controls on the ability to exploit this statistical regularity - whereas we did not observe any difference between groups in a noise-learning task. However, there are several important differences between the two paradigms. In anchoring tasks, the re-occurring stimulus is typically presented on every trial (in AX discrimination tasks), or it is repeatedly drawn from a small set (Ahissar et al., 2006). Also, the re-occurring stimuli are obviously the same, at least for the control group: either pure tones with the same frequency, or the same pseudo-words. Anchoring experiments finally typically include feedback (but see Banai \& Yifat, 2012). Here, the reoccurring stimulus was only present on a minority of trials, at unpredictable times during the experiment (and never on successive trials). The reoccurring stimulus was not initially discriminable from the non-re-occurring ones, as before learning all sounds sounded like noise. No feedback was provided in the test phase. Thus, there are many potential causes for the different outcomes observed in anchoring versus noise learning. Even though the present experiment was not designed to test directly the anchoring theory, an understanding of what caused the success of dyslexic participants in achieving a learning of noise may help to specify the necessary conditions to observe an anchoring deficit.

The present study therefore adds to a growing literature suggesting that auditory processing in dyslexia is largely normal, even in its finer aspects (at least for a majority of individuals). It furthermore provides little support for more recent hypotheses relative 
to deficits in auditory learning or memory (Ramus \& Ahissar, 2012). While this does not in itself have any direct clinical implications, this is certainly consistent with the little measurable efficacy demonstrated by auditory training programs for dyslexia (Strong, Torgerson, Torgerson, \& Hulme, 2011).

Finally, it should be noted that speech has different statistics than noise: for example, speech has much greater peaks and troughs in its envelope (Drullman, 1995) with particularly deep modulations around $4 \mathrm{~Hz}$ (Houtgast \& Steeneken, 1985).

Perceptual learning of speech-specific features should thus also be investigated in future studies. Nevertheless, given the demanding nature of the noise learning task and its $a$ priori generality, our data strongly suggest that unsupervised learning of complex acoustic features is largely preserved in dyslexic individuals.

\section{ACKNOWLEDGMENTS}

This work was supported by CNRS and the Agence Nationale de la Recherche (grants ANR-06-NEUR-022-01, ANR-06-NEURO-019-01, ANR-08-BLAN-0167-01, ANR-2010-BLAN-1906, ANR-10-LABX-0087, ANR-11-BSV4-014-01, and ANR-110001-02 PSL*). We thank Nadège Villiermet, Sanaa Moukawane, Amélie Lachat, and Mathilde Dausse for their contribution to data collection. 


\section{REFERENCES}

Agus, T. R., Thorpe, S. J., \& Pressnitzer, D. (2010). Rapid formation of robust auditory memories: Insights from noise. Neuron, 66(4), 610-618. doi:

10.1016/j.neuron.2010.04.014

Ahissar, M. (2007). Dyslexia and the anchoring-deficit hypothesis. Trends in Cognitive Sciences, 11(11), 458-465. doi: 10.1016/j.tics.2007.08.015

Ahissar, M., Lubin, Y., Putter-Katz, H., \& Banai, K. (2006). Dyslexia and the failure to form a perceptual anchor. Nature Neuroscience, 9(12), 1558-1564. doi: $10.1038 / \mathrm{nn} 1800$

Amitay, S., Ahissar, M., \& Nelken, I. (2002). Auditory processing deficits in reading disabled adults. Journal of the Association for Research in Otolaryngology, 3(3), 302-320. doi: 10.1007/s101620010093

Banai, K., \& Ahissar, M. (2006). Auditory processing deficits in dyslexia: task or stimulus related? Cerebral Cortex, 16(12), 1718-1728. doi: 10.1093/cercor/bhj107

Banai, K., \& Yifat, R. (2012). Auditory working memory and early reading skills in Hebrew-speaking preschool children. Journal of Basic and Clinical Physiology and Pharmacology, 23(3), 109-115. doi: 10.1515/jbcpp-2012-0034

Demany, L., \& Semal, C. (2008). The role of memory in auditory perception. In W. A. Yost, A. N. Popper \& R. R. Fay (Eds.), Auditory Perception of Sound Sources (pp. 77-113). New York: Springer Verlag. 
Dole, M., Hoen, M., \& Meunier, F. (2012). Speech-in-noise perception deficit in adults with dyslexia: effects of background type and listening configuration. Neuropsychologia, 50(7), 1543-1552. doi: 10.1016/j.neuropsychologia.2012.03.007

Drullman, R. (1995). Temporal envelope and fine structure cues for speech intelligibility. Journal of the Acoustical Society of America, 97(1), 585-592. doi: $10.1121 / 1.413112$

Farmer, M. E., \& Klein, R. M. (1995). The evidence for a temporal processing deficit linked to dyslexia: A review. Psychonomic Bulletin \& Review, 2, 460-493. doi: 10.3758/BF03210983

Frederickson, N., Frith, U., \& Reason, R. (1997). Phonological Assessment Battery. Windsor: nferNELSON.

Gola-Asmussen, C., Lequette, C., Pouget, G., Rouyer, C., \& Zorman, M. (2010). ECLA16+: Évaluation des compétences de lecture chez l'adulte de plus de 16 ans. Grenoble: CeFoCOP/Univerité de Provence Aix-Marseille I - Cognisciences LSE Université Pierre Mendès.

Goswami, U., Gerson, D., \& Astric, L. (2010). Amplitude envelope perception, phonology and prosodic sensitivity in children with developmental dyslexia. Reading and Writing, 23(8), 995-1019. doi: 10.1007/s11145-009-9186-6

Goswami, U., Wang, H. L., Cruz, A., Fosker, T., Mead, N., \& Huss, M. (2011). Language-universal sensory deficits in developmental dyslexia: English, Spanish, and Chinese. Journal of Cognitive Neuroscience, 23(2), 325-337. doi: 10.1162/jocn.2010.21453 
Guttman, N., \& Julesz, B. (1963). Lower limits of auditory periodicity analysis. Journal of the Acoustical Society of America, 35(4), 610. doi: 10.1121/1.1918551

Guttorm, T. K., Leppänen, P. H., Poikkeus, A. M., Eklund, K. M., Lyytinen, P., \& Lyytinen, H. (2005). Brain event-related potentials (ERPs) measured at birth predict later language development in children with and without familial risk for dyslexia. Cortex, 41(3), 291-303. doi: 10.1016/S0010-9452(08)70267-3

Hornickel, J., Chandrasekaran, B., Zecker, S., \& Kraus, N. (2012). Auditory brainstem measures predict reading and speech-in-noise perception in school-aged children. Behavioural Brain Research, 216(2), 597-605. doi: 10.1016/j.bbr.2010.08.051

Houtgast, T., \& Steeneken, H. J. M. (1985). A review of the MTF concept in room acoustics and its use for estimating speech intelligibility in auditoria. Journal of the Acoustical Society of America, 77(3), 1069-1077. doi: 10.1121/1.392224

Kaernbach, C. (1993). Temporal and spectral basis of the features perceived in repeated noise. Journal of the Acoustical Society of America, 94(1), 91-97. doi: $10.1121 / 1.406946$

Kaernbach, C. (2004). The memory of noise. Experimental Psychology, 51(4), 240-248. doi: $10.1027 / 1618-3169.51 .4 .240$

Lefavrais, P. (1967). Test d'Alouette (2ème éd.). Paris: Éditions du Centre de Psychologie Appliquée.

Leppänen, P. H., Hämäläinen, J. A., Salminen, H. K., Eklund, K. M., Guttorm, T. K., Lohvansuu, K. (2010). Newborn brain event-related potentials revealing atypical processing of sound frequency and the subsequent association with later literacy 
skills in children with familial dyslexia. Cortex, 46(10), 1362-1376. doi: 10.1016/j.cortex.2010.06.003

Lindgren, S. D., De Renzi, E., \& Richman, L. C. (1985). Cross-national comparisons of developmental dyslexia in Italy and the United States. Child Development, 56(6), 1404-1417. doi: $10.2307 / 1130460$

Macmillan, N. A., \& Creelman, C. D. (2005). Detection theory: A user's guide (2nd edition). Mahwah, NJ: Lawrence Erlbaum Associates.

Motulsky, H., \& Christopoulos, A. (2004). Fitting models to biological data using linear and nonlinear regression. New York: Oxford University Press.

Oganian, Y., \& Ahissar, M. (2012). Poor anchoring limits dyslexics' perceptual, memory, and reading skills. Neuropsychologia, 50(8), 1895-1905. doi:

10.1016/j.neuropsychologia.2012.04.014

Ramus, F. (2003). Developmental dyslexia: specific phonological deficit or general sensorimotor dysfunction? Current Opinion in Neurobiology, 13(2), 212-218. doi: $10.1016 / \mathrm{S} 0959-4388(03) 00035-7$

Ramus, F., \& Ahissar, M. (2012). Developmental dyslexia: the difficulties of interpreting poor performance, and the importance of normal performance. Cognitive Neuropsychology, 29, 104-122. doi: 10.1080/02643294.2012.677420

Ramus, F., Rosen, S., Dakin, S. C., Day, B. L., Castellote, J. M., White, S. (2003). Theories of developmental dyslexia: insights from a multiple case study of dyslexic adults. Brain, 126(4), 841-865. doi: 10.1093/brain/awg076

Ramus, F., \& Szenkovits, G. (2008). What phonological deficit? Quarterly Journal of Experimental Psychology, 61(1), 129-141. doi: 10.1080/17470210701508822 
Ramus, F., White, S., \& Frith, U. (2006). Weighing the evidence between competing theories of dyslexia. Developmental Science, 9, 265-269. doi: 10.1111/j.14677687.2006.00488.x

Raven, J., Raven, J. C., \& Court, J. H. (1998). Advanced progressive matrices. Oxford: Oxford Psychologists Press.

Rosen, S. (2003). Auditory processing in dyslexia and specific language impairment: Is there a deficit? What is its nature? Does it explain anything? Journal of Phonetics, 31, 509-527. doi: 10.1016/S0095-4470(03)00046-9

Strong, G. K., Torgerson, C. J., Torgerson, D., \& Hulme, C. (2011). A systematic metaanalytic review of evidence for the effectiveness of the 'Fast ForWord' language intervention program. Journal of Child Psychology and Psychiatry, 52(3), 224235. doi: 10.1111/j.1469-7610.2010.02329.x

Szmalec, A., Loncke, M., Page, M. P., \& Duyck, W. (2011). Order or disorder? Impaired Hebb learning in dyslexia. Journal of Experimental Psychology: Learning, Memory, and Cognition, 37(5), 1270-1279. doi: 10.1037/a0023820

Tallal, P. (1980). Auditory temporal perception, phonics, and reading disabilities in children. Brain and Language, 9(2), 182-198. doi: 10.1016/0093-934X(80)90139$\mathrm{X}$

Vellutino, F. R., Fletcher, J. M., Snowling, M. J., \& Scanlon, D. M. (2004). Specific reading disability (dyslexia): what have we learned in the past four decades? Journal of Child Psychology and Psychiatry, 45(1), 2-40. doi: 10.1046/j.00219630.2003.00305.x 
Wagner, R. K., \& Torgesen, J. K. (1987). The nature of phonological processing and its causal role in the acquisition of reading skills. Psychological Bulletin, 101, 192212. doi: 10.1037/0033-2909.101.2.192

Wechsler, D. (2000). WAIS-III: Echelle de l'intelligence de Wechsler pour adultes (3ème edition). Paris: Les Éditions du Centre de Psychologie Appliquée.

White, S., Frith, U., Milne, E., Rosen, S., Swettenham, J., \& Ramus, F. (2006). A double dissociation between sensorimotor impairments and reading disability: A comparison of autistic and dyslexic children. Cognitive Neuropsychology, 23(5), 748-761. doi: 10.1080/02643290500438607

White, S., Milne, E., Rosen, S., Hansen, P., Swettenham, J., Frith, U. (2006). The role of sensorimotor impairments in dyslexia: a multiple case study of dyslexic children. Developmental Science, 9(3), 237-255. doi: 10.1111/j.1467-7687.2006.00483.x

World Health Organization. (2011). International statistics classification of diseases and related health problems (10th revision, 4th edition ed.). Geneva: World Health Organization. 


\title{
FOOTNOTES
}

\author{
${ }^{1}$ Due to a technical error, one of the non-dyslexic participants was presented in \\ their final block with one of the RefRNs they had already heard instead of the sixth of \\ their RefRNs. There was no obvious difference in performance for that block so it was \\ included in the results. \\ ${ }^{2}$ The half-life is one of the three parameters of the fitted exponential functions and \\ represented the number of trials in which the hit rates (or false-alarm rates) got halfway \\ closer to their asymptotes.
}


1

2

3

4

5

6

7

8

9

10

11

12

14

15

16

17

18

19

20

21

22

23

24

25

26

27

28

29

30

31

32

33

34

35

36

37

38

39

40

41

42

43

44

45

46

47

48

49

50

51

52

53

54

55

56

57

58

59

60

Table 1. A summary of the blocks presented to each listener, in order.

\begin{tabular}{cccccc}
\hline \#repetitions & $\begin{array}{c}\text { Stimulus } \\
\text { duration } \\
(\mathrm{sec})\end{array}$ & RefRN & RN & $\mathrm{N}$ & \#blocks \\
\hline 10 & 5.0 & - & 5 & 5 & 1 \\
4 & 2.0 & - & 20 & 20 & 1 \\
3 & 1.5 & - & 20 & 20 & 1 \\
2 & 1.0 & - & 20 & 20 & 1 \\
2 & 1.0 & 20 & 20 & 40 & 6 \\
\hline
\end{tabular}


Table 2. A summary of participants' demographic characteristics and performance on the diagnostic battery.

\begin{tabular}{|c|c|c|c|c|c|c|c|c|}
\hline & \multicolumn{4}{|c|}{ Controls $(\mathrm{N}=18)$} & \multicolumn{4}{|c|}{ Dyslexics $(\mathrm{N}=18)$} \\
\hline & Mean & SD & Min. & Max. & Mean & SD & Min. & Max. \\
\hline $\begin{array}{l}\text { Age } \\
\text { (years) }\end{array}$ & 24.01 & 3.93 & 19.00 & 32.01 & 23.54 & 5.24 & 17.85 & 36.46 \\
\hline $\begin{array}{l}\text { Non-verbal } \\
\text { IQ }\end{array}$ & 115.06 & 13.88 & 84.00 & 135.00 & 109.61 & 12.39 & 90.00 & 131.00 \\
\hline $\begin{array}{l}\text { Reading } \\
\text { fluency } \\
\text { (z-score) }\end{array}$ & 1.50 & 1.16 & -0.60 & 4.21 & -1.70 & 0.55 & -2.86 & -1.10 \\
\hline $\begin{array}{l}\text { Orthographic } \\
\text { choice } \\
\text { (items } / \mathrm{sec} \text { ) }\end{array}$ & 0.46 & 0.21 & 0.00 & 0.79 & 0.19 & 0.09 & 0.00 & 0.33 \\
\hline $\begin{array}{l}\text { RAN } \\
\text { composite } \\
\text { (sec) }\end{array}$ & 47.69 & 7.23 & 6.00 & 18.00 & 6.61 & 2.59 & 2.00 & 11.00 \\
\hline $\begin{array}{l}\text { Digit-span } \\
\text { scaled score }\end{array}$ & 10.56 & 3.31 & 6.00 & 18.00 & 6.61 & 2.59 & 2.00 & 11.00 \\
\hline $\begin{array}{l}\text { Spoonerisms } \\
\text { (item } / \mathrm{sec} \text { ) }\end{array}$ & 0.11 & 0.05 & 0.03 & 0.25 & 0.05 & 0.03 & 0.01 & 0.13 \\
\hline
\end{tabular}




\section{FIGURE CAPTIONS}

Figure 1. A visual illustration of the repetition-detection task performed by listeners. The figure shows one possible ordering of the different conditions, with schematic spectrograms of the stimuli and the correct responses ("yes" or "no") for each trial. Listeners were trained to distinguish 1-s long noises ("N"; trials 1, 5, and 7) from noises that consisted of two abutting presentations of the same 0.5-s noise token (repeated noises or "RN"; trials 2 and 4). Unbeknownst to the listeners, half of the repeated noises were identical across different trials ("RefRN"; trials 3 and 6). Therefore, RN trials include a within-trial repetition (the two halves of the spectrograms are identical within a trial), but no across-trial repetition (spectrograms differ across trials). In contrast, the RefRN trials have both within- and across-trial repetitions (the spectrograms are the same throughout). For the $\mathrm{N}$ trials, there is no repeat, within nor across trials.

Figure 2. The training data, including mean hit rates for $\mathrm{RN}$ and false-alarm rates for $\mathrm{N}$ for the dyslexic listeners and the control group. The error bars are $95 \%$ confidence intervals centered on the mean.

Figure 3. Mean $d^{\prime}$ sensitivity for RN and RefRN for the two groups of listeners. Error bars are $95 \%$ confidence intervals centered on the mean.

Figure 4. The time course of average hit rates for RefRN, RN and false-alarm rates for $\mathrm{N}$ for the dyslexic listeners and the control group. Each point is calculated as the average 
proportion of "repeated" responses across all blocks for the $n^{\text {th }}$-presented trial of a given type of stimulus.

Figure 5. The same data shown in Figure 4 split into blocks for which learning was observed (top panel) and blocks for which learning was not observed (lower panel). Learning was judged to have occurred in a block if the number of repetitions reported for RefRN significantly exceeded those for RN, as measured by a Fisher exact test without correction for multiple comparisons. 
Figure 1. A visual illustration of the repetition-detection task performed by listeners. The figure shows one possible ordering of the different conditions, with schematic spectrograms of the stimuli and the correct responses ("yes" or "no") for each trial. Listeners were trained to distinguish 1-s long noises ("N"; trials 1, 5, and 7) from noises that consisted of two abutting presentations of the same 0.5 -s noise token (repeated noises or "RN"; trials 2 and 4). Unbeknownst to the listeners, half of the repeated noises were identical across different trials ("RefRN"; trials 3 and 6). Therefore, RN trials include a within-trial repetition (the two halves of the spectrograms are identical within a trial), but no across-trial repetition (spectrograms differ across trials). In contrast, the RefRN trials have both within- and across-trial repetitions (the spectrograms are the same throughout). For the $\mathrm{N}$ trials, there is no repeat, within nor across trials. $100 \times 62 \mathrm{~mm}(300 \times 300 \mathrm{DPI})$ 


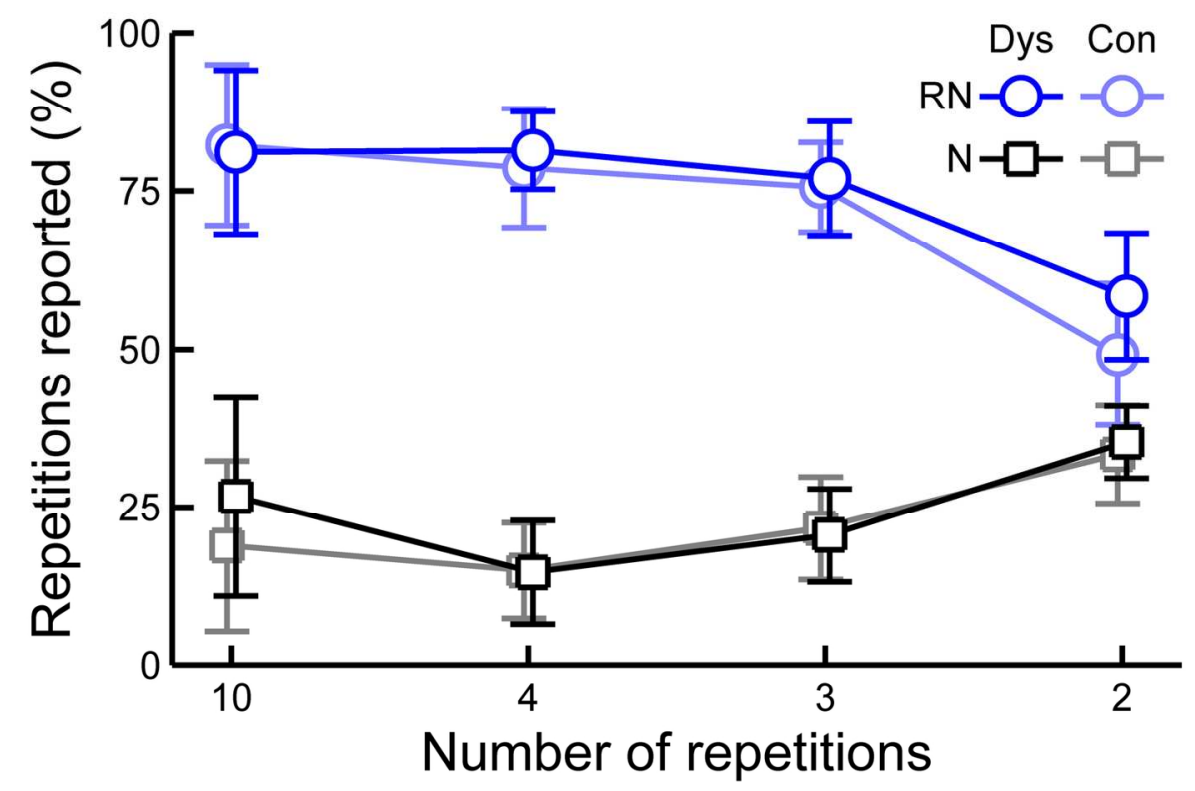

Figure 2. The training data, including mean hit rates for $\mathrm{RN}$ and false-alarm rates for $\mathrm{N}$ for the dyslexic listeners and the control group. The error bars are $95 \%$ confidence intervals centered on the mean. 
1

2

3

4

5

6

7

8

9

10

11

12

13

14

15

16

17

18

19

20

21

22

23

24

25

26

27

28

29

30

31

32

33

34

35

36

37

38

39

40

41

42

43

44

45

46

47

48

49

50

51

52

53

54

55

56

57

58

59

60

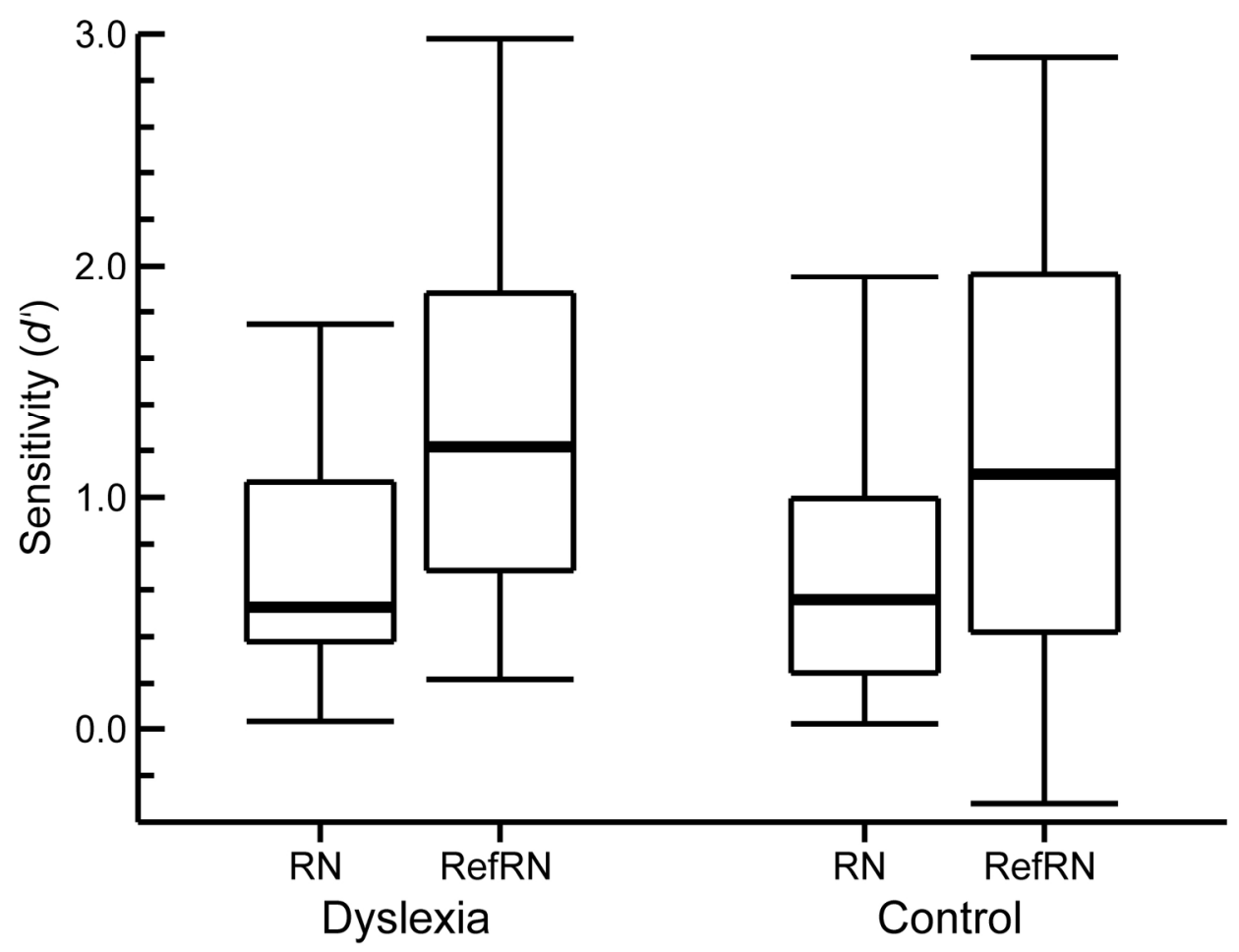

Figure 3. Mean d' sensitivity for RN and RefRN for the two groups of listeners. Error bars are $95 \%$ confidence intervals centered on the mean. $90 \times 67 \mathrm{~mm}(600 \times 600 \mathrm{DPI})$ 


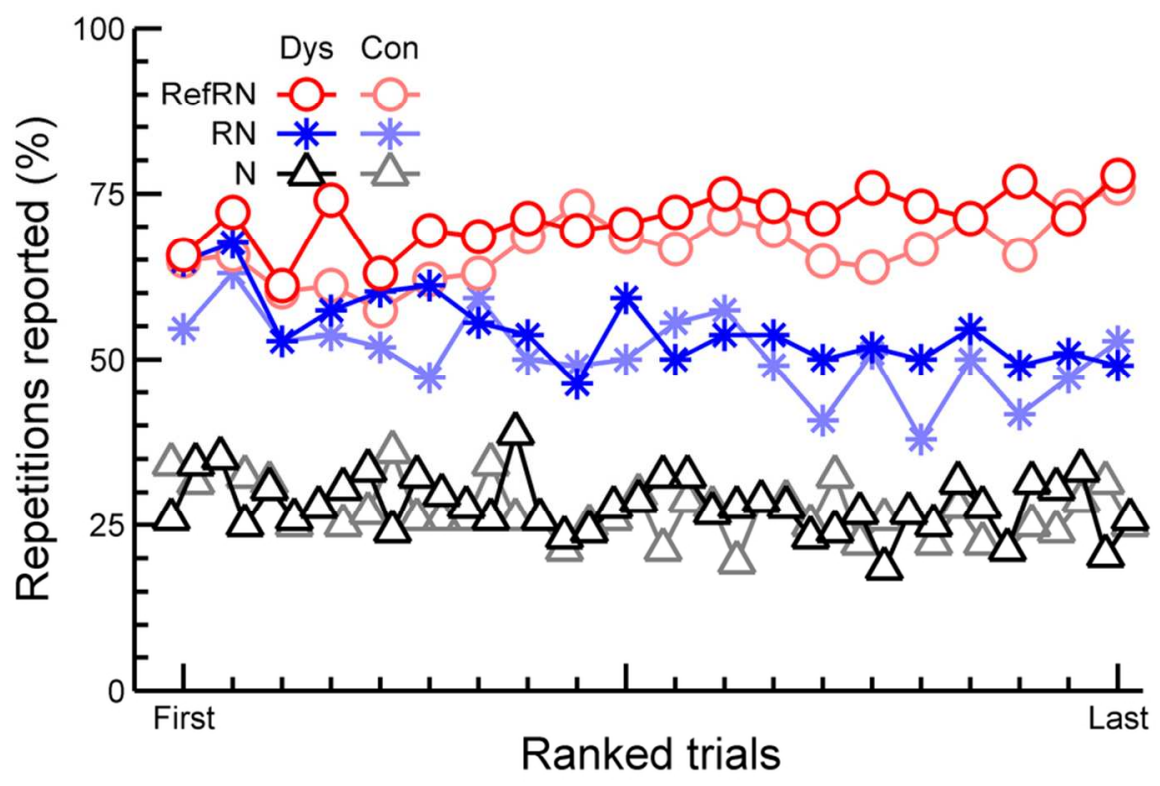

Figure 4. The time course of average hit rates for RefRN, RN and false-alarm rates for $\mathrm{N}$ for the dyslexic listeners and the control group. Each point is calculated as the average proportion of "repeated" responses across all blocks for the nth-presented trial of a given type of stimulus. 

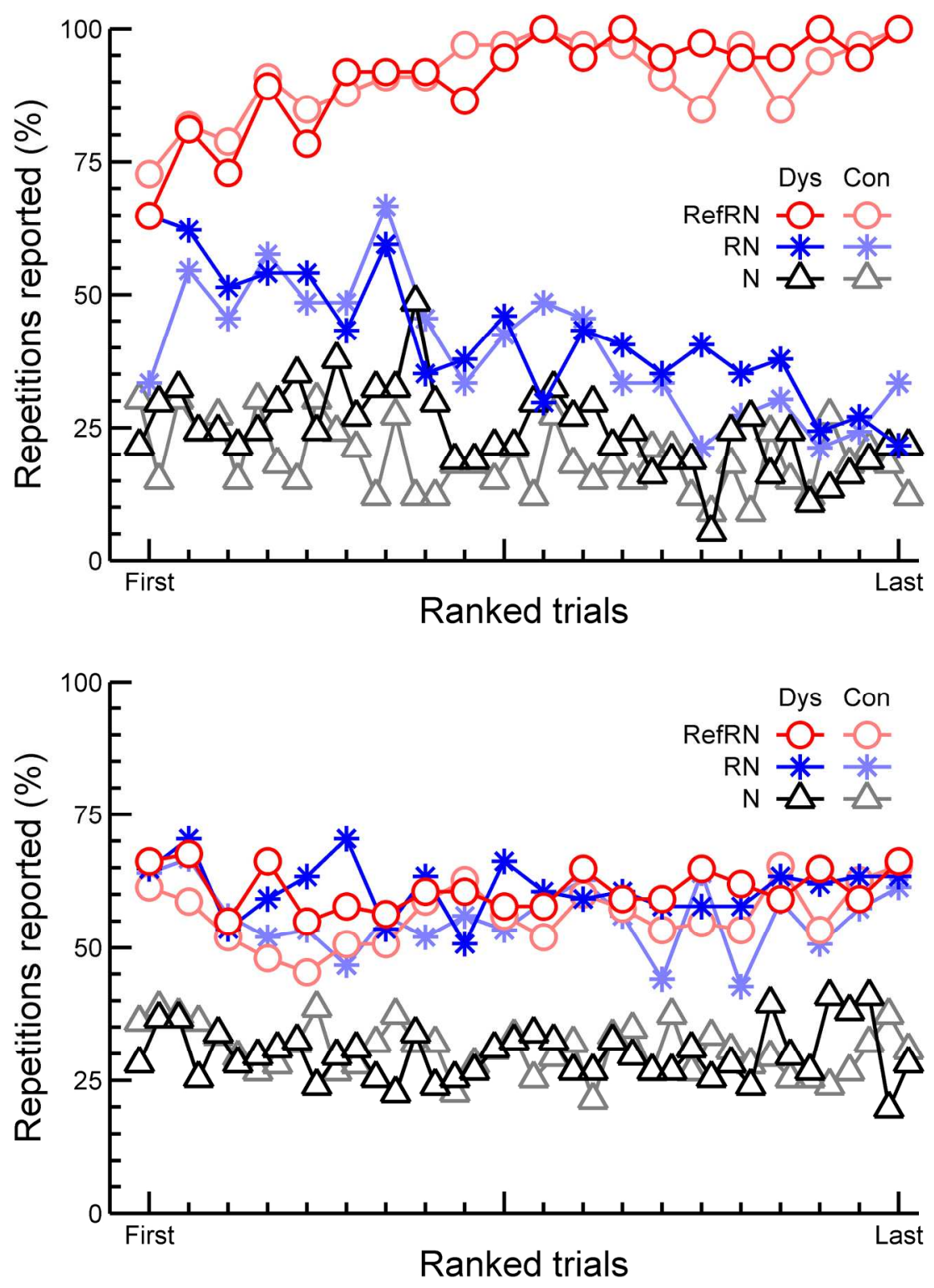

Figure 5. The same data shown in Figure 4 split into blocks for which learning was observed (top panel) and blocks for which learning was not observed (lower panel). Learning was judged to have occurred in a block if the number of repetitions reported for RefRN significantly exceeded those for RN, as measured by a Fisher exact test without correction for multiple comparisons. $180 \times 224 \mathrm{~mm}(300 \times 300$ DPI $)$ 